\title{
Design of Robust Internal Model Control for Multivariate Descriptor Systems Based on Time-scale Transformation
}

\author{
Beiyan Jiang *, Meiying Jiang, Yuming Zhang, Xinghan Du, Yan Huang and Wu Cai \\ Beijing University of Chemical Technology, Beisanhuan East Road 15, Chaoyang District, Beijing, PR China \\ ${ }^{*}$ Corresponding author
}

\begin{abstract}
This paper deals with a design problem of robust internal model control (IMC) for multivariate descriptor linear systems with more inputs than outputs, which establishes a possible way to design and analyze descriptor systems. The main idea of the method is that the given system is first decomposed into slow and fast subsystems. And then the internal model controller and dynamic compensator are designed for the two subsystems respectively. For the sake of implementation, timescale transformation is introduced. In virtue of uncertainties always exist in practical system, a robust controller is designed for improving the stability and the performance. Finally, an example is adopted to demonstrate the validity and feasibility of the new method. It is shown that the proposed strategy not only guarantees control effect of the closed-loop system, but also simultaneously eliminates the impulsive behavior of the fast subsystem.
\end{abstract}

Keywords-multivariate descriptor system; internal model control; transfer function; robust performance

\section{INTRODUCTION}

In practice, a lot of dynamic systems are described by descriptor system models for their physical variables cannot be chosen as state variables in a natural way. Due to their theoretical importance and extensive applications in modeling and control of economics [1], biological systems [2], and chemical engineering [3], descriptor systems have received considerable interests since they were first mentioned in [4]. In recent years, the problems of stabilization, optimal control, impulse elimination, and observer design for descriptor systems have been extensively studied, see references [5-6]. Apparently, both of these methods have their own advantages, but they also have their inherent limitations to solving problems such as too complicated for practical interests, failed to yield optimal controllers, and so on. Hence, the problem of robust control of descriptor systems has not been fully studied in the literature and still remains open.

IMC has been widely employed in process control in the last twenty years for its advantages of simple design, excellent robustness and good control performance, see [7]. But there are very few works on IMC of descriptor systems has been reported for the controllers must be designed so that the closed-loop descriptor system is not only robustly stable, but also regular and impulse-free while part maybe associated with the irregularity of system transfer matrices. Therefore, constructing rational control structures and extending IMC method to descriptor systems truly possess of both theoretical interest and practical significance.

In this paper, we attempt to deal with a design problem of robust internal model control for multivariate descriptor linear system with more inputs than outputs. The main idea of the method is first decompose the given system into slow and fast subsystems, and then the internal model controller and dynamic compensator are designed for the two subsystems respectively. For the sake of implementation, time-scale transformation is introduced. In virtue of uncertainties always exist in practical systems, a robust controller with PI structure is designed for improving the stability and the performance.

\section{PROBLEM STATEMENT AND PRELIMINARIES}

Consider a class of linear multivariate descriptor systems described by the following linear differential equation:

$$
\left\{\begin{array}{l}
\boldsymbol{E} \dot{\boldsymbol{x}}(t)=\boldsymbol{A} \boldsymbol{x}(t)+\boldsymbol{B} \boldsymbol{u}(t)+\boldsymbol{B}_{1} \boldsymbol{w}(t) \\
\boldsymbol{y}(t)=\boldsymbol{C} \boldsymbol{x}(t)
\end{array}\right.
$$

where $\boldsymbol{x}(t) \in R^{n}, \quad \boldsymbol{u}(t) \in R^{m}, \boldsymbol{w}(t)$ and $\boldsymbol{y}(t) \in R^{p}$ are, respectively, the state vector, the input vector, the disturbance vector and the output vector; $\boldsymbol{A} \in R^{n \times n}, \boldsymbol{B} \in R^{n \times m}, \boldsymbol{B}_{1} \in R^{n \times m}$ and $\boldsymbol{C} \in R^{p \times n} \quad(m, p<n)$ are known nonsingular constant matrices. The matrix $\boldsymbol{E} \in R^{n \times n}$ may be singular with $\operatorname{rank}(\boldsymbol{E})=n_{1}<n$. To guarantee that system (1) has an unique solution, we hereafter assume that matrix pair $(\boldsymbol{E}, \boldsymbol{A})$ is regular.

We first give the following lemmas, which are essential in establishing the main results of this work.

Lemma 1 Let $\boldsymbol{G}(s)$ be the transfer matrix of system (1). Then

$$
\boldsymbol{G}(s)=\boldsymbol{C}(s \boldsymbol{E}-\boldsymbol{A})^{-1} \boldsymbol{B}=\tau \hat{\boldsymbol{C}}(\tau \boldsymbol{I}-\hat{\boldsymbol{E}})^{-1} \hat{\boldsymbol{B}}=\hat{\boldsymbol{G}}(\tau)
$$

where $\hat{\boldsymbol{E}}=(a \boldsymbol{E}+\boldsymbol{A})^{-1} \boldsymbol{E}, \quad \hat{\boldsymbol{B}}=(a \boldsymbol{E}+\boldsymbol{A})^{-1} \boldsymbol{B}, \hat{\boldsymbol{C}}=-\boldsymbol{C}$, $\tau=\frac{1}{s+a}$, and $a$ satisfies $|a \boldsymbol{E}+\boldsymbol{A}| \neq 0 . \tau$ is called the time- 
scale transformation for descriptor systems. It implies a relationship between infinite and finite.

From [1], there exists two nonsingular matrices $\boldsymbol{P}_{1}$ and $\boldsymbol{P}_{2}$ such that the system (1) can be transformed into:

$$
\begin{array}{r}
\dot{\boldsymbol{x}}_{1}(t)=\boldsymbol{A}_{1} \boldsymbol{x}_{1}(t)+\boldsymbol{B}_{1} \boldsymbol{u}(t), \quad \boldsymbol{y}_{1}(t)=\boldsymbol{C}_{1} \boldsymbol{x}_{1}(t) \\
\boldsymbol{N} \dot{\boldsymbol{x}}_{2}(t)=\boldsymbol{x}_{2}(t)+\boldsymbol{B}_{2} \boldsymbol{u}(t), \quad \boldsymbol{y}_{2}(t)=\boldsymbol{C}_{2} \boldsymbol{x}_{2}(t) \\
\boldsymbol{y}(t)=\boldsymbol{y}_{1}(t)+\boldsymbol{y}_{2}(t)
\end{array}
$$

where, $\boldsymbol{x}_{1}(t) \in \boldsymbol{R}^{n_{1}}, \boldsymbol{x}_{2}(t) \in \boldsymbol{R}^{n_{2}}, n_{1}+n_{2}=n, \quad \boldsymbol{N} \in \boldsymbol{R}^{n_{2} \times n_{2}}$ is nilpotent whose nilpotent denoted by $h$. Then the transfer function matrix of system (1) can be expressed as:

$$
\begin{aligned}
\boldsymbol{G}(s) & =\boldsymbol{C}_{1}\left(s \boldsymbol{I}-\boldsymbol{A}_{1}\right)^{-1} \boldsymbol{B}_{1}+\boldsymbol{C}_{2}(s \boldsymbol{N}-\boldsymbol{I})^{-1} \boldsymbol{B}_{2} \\
& =\boldsymbol{G}_{s}(s)+\boldsymbol{G}_{q}(s)
\end{aligned}
$$

where $\boldsymbol{G}_{s}(s)$ is strictly proper, called slow subsystem determined by (3-a); $\boldsymbol{G}_{q}(s)$ is polynomial, called fast subsystem determined by (3-b).

\section{CONTROller Design SCHEME}

From formula (4), the control problem of descriptor linear system (1) is converted into that of the slow and the fast subsystems. The improved IMC design scheme used in the system is illustrated on Figure 1.

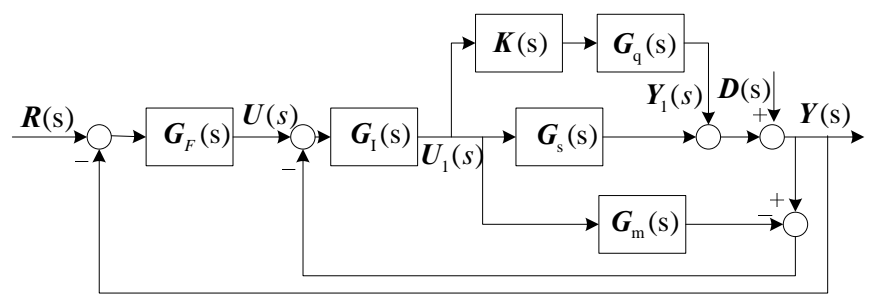

FIGURE I. THE CONTROL STRUCTURE OF SINGULAR SYSTEM

In this scheme, the dynamic compensator $\boldsymbol{K}(s)$ is proposed to address the impulse elimination problem of $\boldsymbol{G}_{q}(s)$ The internal model controller $\boldsymbol{G}_{\mathrm{I}}(s)$, which is designed based on the approximate mode $\boldsymbol{G}_{m}(s)$, is used to suppress disturbance since $\boldsymbol{G}_{s}(s)$. The robust controller $\boldsymbol{G}_{\mathrm{F}}(s)$ is given to guarantee the robust stability of the system. Therefore, combined with robust control and dynamic compensator, the overall IMC scheme represents a master-slave closed-loop system with guaranteed stability and performance.

\section{A. Elimination of Impulses by Dynamic Compensator}

In this subsection we are interested in designing a dynamic compensator for the fast subsystem to be stable and impulsefree. From the preceding description, we know that the transfer function of $\boldsymbol{G}_{q}(s)$ is not proper. Time scale transformation in lemma 2 has been turned out to be an effective way to solve this problem.

In formula (2), let $a=0, \boldsymbol{G}_{q}(s)$ is easily seen to generate the same transfer function

$$
\overline{\boldsymbol{G}}_{q}(s)=-\frac{1}{S} \boldsymbol{G}_{q}\left(\frac{1}{S}\right)
$$

By several transformations, $\overline{\boldsymbol{G}}_{q}(s)$ is illustrated to be strictly proper. Furthermore, we can use the abundant results in linear system theory to study it. We intend to design a controller $\boldsymbol{K}(s)$ to attenuate its influence on system (1). [8] shows that the closed-loop system can be made impulse-free by almost any output feedback if and only if $\boldsymbol{G}_{q}(s)$ is both impulse controllable and observable. To keep the order of the compensator $\boldsymbol{K}(s)$ low, we select $\boldsymbol{K}(s)$ as

$$
\boldsymbol{K}(s)=\boldsymbol{M} \overline{\boldsymbol{G}}_{q}^{*}(0) \boldsymbol{F}_{q}(s)
$$

where, $\boldsymbol{F}_{q}(s)=\operatorname{diag}\left\{(s+1)^{-1}\right\}$ is used to eliminate algebraic loop, $\overline{\boldsymbol{G}}_{q}^{*}(0)$ is the pseudo-inverse of $\overline{\boldsymbol{G}}_{q}(0)$ selected to ensure the subsystem can realize static decoupling and $\boldsymbol{M}=\operatorname{diag}\left\{m_{i}\right\}$ is a constant matrix. For a given positive $\varepsilon$, $\|\boldsymbol{M}\|$ is small enough to guarantee the following formula always be true

$$
\left\|\overline{\boldsymbol{G}}_{q}(s) \boldsymbol{K}(s)\right\|<\varepsilon, \quad \forall s>0
$$

In Figure 1, the output of fast subsystem is

$$
\boldsymbol{Y}_{1}(s)=\boldsymbol{G}_{U_{1} Y_{1}}(s) \boldsymbol{U}_{1}(s)
$$

where $\boldsymbol{G}_{U_{1} Y_{1}}(s)=\overline{\boldsymbol{G}}_{q}(s) \boldsymbol{K}(s)$

Combining formulas (6) and (8), yields

$$
\boldsymbol{Y}_{1}(0)=\boldsymbol{M} \boldsymbol{U}_{1}(0)
$$

$\forall s>0$, we can enable $\boldsymbol{Y}_{1}(s)$ is small enough to be regarded as the system uncertainties or be ignored by the reasonable design of controller parameters matrix $\boldsymbol{M}$.

\section{B. IMC Controller}

From reference [1], the controller $\boldsymbol{G}_{\mathrm{I}}(s)$ can be designed as

$$
\boldsymbol{G}_{I}(s)=\boldsymbol{G}_{m}^{*}(s) \boldsymbol{G}_{m+}(s) \boldsymbol{F}(s)
$$


In Figure 1, it is evident to

$$
\boldsymbol{Y}(s)=\boldsymbol{G}_{U Y}(s) \boldsymbol{U}(s)+\boldsymbol{G}_{D Y}(s) \boldsymbol{D}(s)
$$

$$
\text { where } \quad \boldsymbol{G}_{U Y}(s)=\frac{\boldsymbol{G}_{p}(s) \boldsymbol{G}_{I}(s)}{\boldsymbol{I}+\left[\boldsymbol{G}_{p}(s)-\boldsymbol{G}_{m}(s)\right] \boldsymbol{G}_{I}(s)}
$$$$
\boldsymbol{G}_{D Y}(s)=\frac{\boldsymbol{I}-\boldsymbol{G}_{m}(s) \boldsymbol{G}_{I}(s)}{\boldsymbol{I}+\left[\boldsymbol{G}_{p}(s)-\boldsymbol{G}_{m}(s)\right] \boldsymbol{G}_{I}(s)}, \boldsymbol{G}_{p}(s)=\boldsymbol{G}_{s}(s)+\overline{\boldsymbol{G}}_{q}(s) \boldsymbol{K}(s)
$$

Assume $\varepsilon<<1$, formula (11) is written as:

$$
\boldsymbol{Y}(s)=\boldsymbol{G}_{m+}(s) \boldsymbol{F}(s) \boldsymbol{U}(s)+\left(\boldsymbol{I}-\boldsymbol{G}_{m+}(s) \boldsymbol{F}(s)\right) \boldsymbol{D}(s)
$$

Through analysis formula (12), the internal model controller is adopted to resist disturbance. Hence, the filter parameter $\lambda_{i}$ should be chosen to minimize $\boldsymbol{I}-\boldsymbol{G}_{m+}(s) \boldsymbol{F}(s)$.

\section{Robust Controller}

Assume that the uncertainties can be described as

$\Delta \boldsymbol{A}=\boldsymbol{P}_{1}^{-1} \operatorname{diag}\left\{\beta_{1} \boldsymbol{I}, 0\right\} \boldsymbol{P}_{2}^{-1}, \Delta \boldsymbol{B}=\boldsymbol{P}_{1}^{-1} \operatorname{diag}\left\{\beta_{2} \boldsymbol{I}, 0\right\}$,

$$
\Delta \boldsymbol{C}=\operatorname{diag}\left\{\beta_{3} \boldsymbol{I}, 0\right\} \boldsymbol{P}_{2}^{-1}
$$

where $\left\|\operatorname{diag}\left\{\beta_{i} \boldsymbol{I}, 0\right\}\right\| \leq \boldsymbol{I}, \quad i=1,2,3$.

Substitute formulas (10) into formula (11), we have

$$
\boldsymbol{G}_{U Y}(s)=\frac{\boldsymbol{G}_{m+}(s) \boldsymbol{F}(s)}{\frac{\boldsymbol{G}_{m}(s)}{\boldsymbol{G}_{p}(s)}+\frac{\left[\boldsymbol{G}_{p}(s)-\boldsymbol{G}_{m}(s)\right]}{\boldsymbol{G}_{p}(s)} \boldsymbol{G}_{m+}(s) \boldsymbol{F}(s)}
$$

Let $\boldsymbol{\varphi}(s)=\frac{\boldsymbol{G}_{p}(s)-\boldsymbol{G}_{m}(s)}{\boldsymbol{G}_{p}(s)}$, it represents the multiplicative uncertainties. $\varphi(s)$ is bounded, i.e. $\|\varphi(s)\| \leq \alpha$. Then formula (9) can be rewritten as

$$
\boldsymbol{G}_{U Y}(s)=\frac{\boldsymbol{G}_{m+}(s) \boldsymbol{F}(s)}{\boldsymbol{I}+\boldsymbol{\varphi}(s)\left[\boldsymbol{I}-\boldsymbol{G}_{m+}(s) \boldsymbol{F}(s)\right]}
$$

Let $\boldsymbol{\delta}(s)=-\frac{\boldsymbol{\varphi}(s)\left[\boldsymbol{I}-\boldsymbol{G}_{m+}(s) \boldsymbol{F}(s)\right]}{\boldsymbol{I}+\boldsymbol{\varphi}(s)\left[\boldsymbol{I}-\boldsymbol{G}_{m+}(s) \boldsymbol{F}(s)\right]}$, and $\boldsymbol{\delta}(s)$ is also bounded. Formula (14) can be further simplified as

$$
\boldsymbol{G}_{U Y}(s)=[1+\boldsymbol{\delta}(s)] \boldsymbol{G}_{m+}(s) \boldsymbol{F}(s)
$$

According to above deduction, if $\boldsymbol{G}_{p}(s)=\boldsymbol{G}_{m}(s)$, we have $\boldsymbol{\varphi}(s)=0, \boldsymbol{\delta}(s)=0$, and $\boldsymbol{G}_{U Y}(s)=\boldsymbol{G}_{m+}(s) \boldsymbol{F}(s)$. If we consider the influence of $\boldsymbol{G}_{q}(s)$ and parameter uncertainties, the modeling error produced by them can be represented by the variation of $\boldsymbol{G}_{m+}(s) \boldsymbol{F}(s)$. Then the inner loop of IMC can be equivalent to a system $\overline{\boldsymbol{F}}(s)$ whose nominal transfer function is $\boldsymbol{G}_{m+}(s) \boldsymbol{F}(s)$, and whose uncertainty model is multiplicative perturbation, i.e. $\overline{\boldsymbol{F}}(s)=[1+\boldsymbol{\delta}(s)] \boldsymbol{G}_{m+}(s) \boldsymbol{F}(s)$. Hence, Figure 1 can be equivalent to Figure 2 .

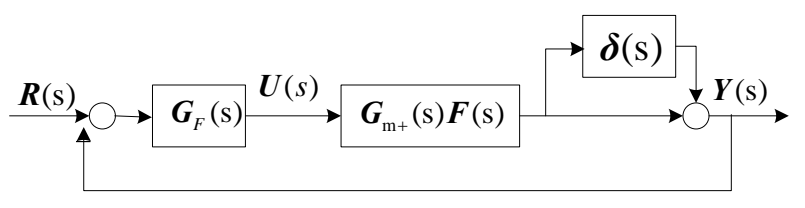

FIGURE II. THE EQUIVALENCE STRUCTURE OF FIG. 1

Now robust controller $\boldsymbol{G}_{F}(s)$ is much easier to design. Next, we will give a theorem to illustrate the design method of $\boldsymbol{G}_{F}(s)$.

Theorem 3.1 Consider the feedback control system shown in Figure 2, controller $\boldsymbol{G}_{F}(s)$ provides robust stability if and only if

$$
\|\boldsymbol{\delta}(s) \overline{\boldsymbol{G}}(s)\|<1
$$

where $\overline{\boldsymbol{G}}(s)=\frac{\boldsymbol{G}_{m+}(s) \boldsymbol{F}(s) \boldsymbol{G}_{F}(s)}{\boldsymbol{I}+\boldsymbol{G}_{m+}(s) \boldsymbol{F}(s) \boldsymbol{G}_{F}(s)}$.

Robust controller $\boldsymbol{G}_{F}(s)$ is designed to realize the tracking performance and eliminate stable state error. We choose PI as the controller structure of $\boldsymbol{G}_{F}(s)$, expressed as:

$$
\boldsymbol{G}_{F}(s)=k_{p}\left(1+\frac{1}{s}\right)
$$

where $k_{p}$ is a constant, which is determined to achieve the expected control performance.

\section{SimUlation}

Let us considering the following not square singular system

$$
\begin{gathered}
\left\{\begin{array}{l}
\boldsymbol{E} \dot{\boldsymbol{x}}(t)=\boldsymbol{A} \boldsymbol{x}(t)+\boldsymbol{B} \boldsymbol{u}(t) \\
\boldsymbol{y}(t)=\boldsymbol{C} \boldsymbol{x}(t)
\end{array}\right. \\
\text { where, } \boldsymbol{E}=\left[\begin{array}{cccc}
1 & 0 & 0 & 0 \\
-1 & 1 & -1 & 0 \\
-1 & 0 & 0 & 0 \\
0 & 0 & 1 & 0
\end{array}\right], \boldsymbol{A}=\left[\begin{array}{cccc}
-1 & 0 & 0 & 0 \\
2 & -2 & 0 & -1 \\
1 & 0 & -1 & 0 \\
0 & 0 & 0 & 1
\end{array}\right], \\
\boldsymbol{B}=\left[\begin{array}{llll}
1 & 0 & 0 & 0 \\
0 & 0 & 1 & 1
\end{array}\right]^{T}, \quad \boldsymbol{C}=\left[\begin{array}{llll}
0 & 1 & 2 & 1
\end{array}\right]
\end{gathered}
$$


The transfer functions of slow sub-system $\boldsymbol{G}_{s}(s)$ and fast sub-system $\boldsymbol{G}_{q}(s)$ are:

$$
\boldsymbol{G}_{s}(s)=\left[\begin{array}{c}
\frac{1}{s+1} \\
\frac{1}{s+2}
\end{array}\right]^{T}, \boldsymbol{G}_{q}(s)=\left[\begin{array}{c}
s+2 \\
s+1
\end{array}\right]^{T}, \overline{\boldsymbol{G}}_{q}(s)=\left[\begin{array}{c}
-\frac{2 s+1}{s^{2}} \\
-\frac{s+1}{s^{2}}
\end{array}\right]^{T}
$$

From formulas (6), (10) and (15), the controllers are given as:

$$
\begin{aligned}
& \boldsymbol{K}(s)=-\frac{1}{s+1}\left[\begin{array}{c}
\frac{m_{1}\left(2 s^{3}+s^{2}\right)}{5 s^{2}+6 s+2} \\
\frac{m_{2}\left(s^{3}+s^{2}\right)}{5 s^{2}+6 s+2}
\end{array}\right], \boldsymbol{G}_{\mathrm{I}}(s)=\left[\begin{array}{c}
\frac{s^{3}+5 s^{2}+8 s+4}{(\lambda s+1)\left(2 s^{2}+6 s+5\right)} \\
\frac{s^{3}+4 s^{2}+5 s+2}{(\lambda s+1)\left(2 s^{2}+6 s+5\right)}
\end{array}\right], \\
& \boldsymbol{G}_{F}(s)=\frac{k_{p}(s+1)}{s}
\end{aligned}
$$

Given the step input $r=1$ and disturbance $d=0.4$, the optimum parameters of controllers searched by Gas are found as $\lambda=1.5847, k_{p}=1.4236, m_{1}=0.3130$ and $m_{2}=0.2393$.

To verify the effective of the proposed methods, the proposed method controlled, and robust systems, the performance of the controllers for set point responses and are shown in the following simulations for both model matching and parametric uncertainties.

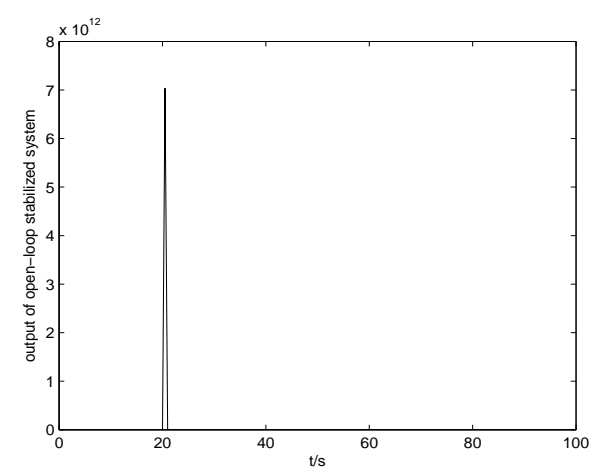

FIGURE III. OUTPUT RESPONSES OF UNCONTROLLED SYSTEM

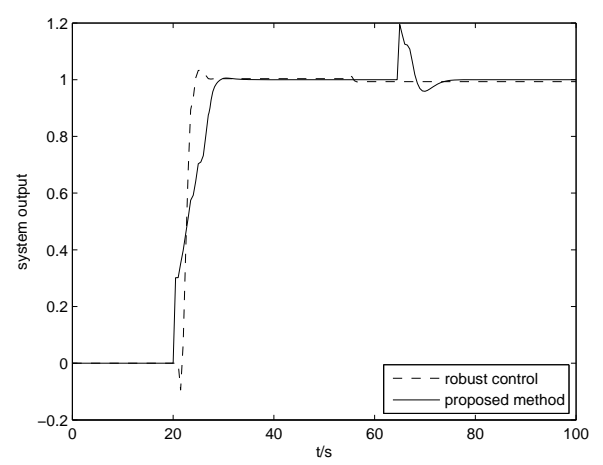

FIGURE IV. OUTPUT RESPONSES WHEN MODEL MATCHES COMPLETELY
In order to validate the robustness of the proposed method, we assume uncertainties exist only in $\|\Delta \boldsymbol{C}\| \leq 0.2$ for calculating easily. Simulation results are shown in the following figures.

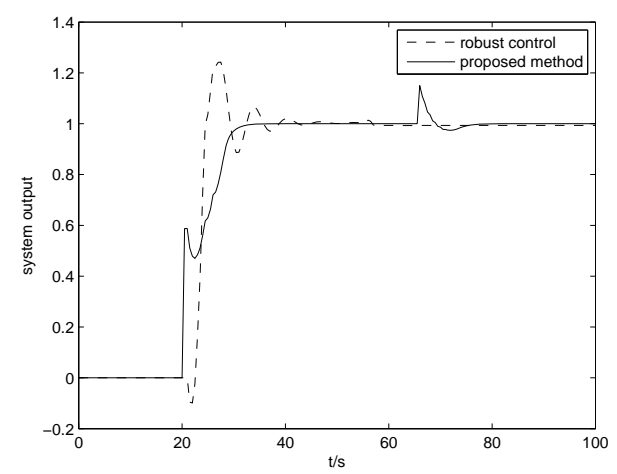

FIGURE V. OUTPUT RESPONSES WHEN UNCERTAINTIES EXIST

Simulation results in Figures 3-5 confirm that the proposed controllers indeed have better tracking performance and faster response speed than classical robust control method whether the uncertainties exist or not for error ranges are smaller and converge to zero faster.

\section{CONCLUSIONS}

In this paper, we broke the limiting of IMC is only viable for nonsingular systems, established a constructive method for designing and analyzing descriptor systems. A robust IMC method was first proposed for multivariate descriptor systems with more inputs than outputs. The control object was first decomposed into separate slow and fast subsystems of lower dimensions. Then the internal model controller and dynamic compensator are designed for the two subsystems respectively. Time-scale transformation was employed to ensure the implement of fast subsystem control and a robust controller was designed for improving the stability and the performance. Example demonstrated that the proposed strategy not only avoids complicated computations for studying a relatively simpler restricted equivalent form of complex systems, but also guarantees control effect by converting the control design for high-order system into that of two lower order subsystems when the impulsive behavior of the fast subsystem is eliminated.

\section{ACKNOWLEDGMENT}

This work was partially supported by the Fundamental Research Funds for the Central Universities (Grant ZY1619) and partly by the Automation Institute Beijing University of Chemical Technology. The authors are grateful to the anonymous reviewers for their valuable recommendations.

\section{REFERENCES}

[1] D. G. Luenberger and A. Arbel, Singular dynamic Leontief systems, Econometrica, 45(4), (1977) 991-995. 
[2] A. James, Y. C. Chang, P. Antonis, Model decomposition and reduction tools for large-scale networks in systems biology, Automatica, 47(6), (2011) 1165-1174.

[3] D. Cobb, Controllability, observability, and duality in singular systems, IEEE Trans. Automat. Contr., 29(12), (1984) 1076- 1082.

[4] S. P. Singh and R. W. Liu, Existence of state equation representation of linear large-scale dynamic systems, IEEE Trans. Circuit Theor., 20(5), (1973) 239-246.

[5] D. N. Shields, Observer design and detection for nonlinear descriptor systems, Int. J. Control, 67(2), (1997) 153-168.

[6] D. H. Wang and P. Bao, Robust impulse control of uncertain singular systems by decentralized output feedback, IEEE Trans. Automat. Contr., 45(3), (2000) 500-505.

[7] C. E. Garcia and M. Morari, Internal Model Control. 1. A Unifying Review and Some New Result, Ind. Eng. Chem. Res. Design and Development, 21(2), (1982) 308-323.

[8] R. Y. Yu and D. H. Wang, Structural properties and poles assignability of LTI singular systems under output feedback, Automatica, 39(4), (2003) 685-692 